\title{
The Characterization of Poly n-Vinyl Pyrrolidone-Polyvinyl Acetate (PVP-PVAc) Copolymers and Blends by Nuclear Magnetic Resonance Spectroscopy, Fourier Transform Infrared Spectroscopy, and Elemental Analysis
}

\author{
Ronald P. D'Amelia ", Joseph Mancuso, William Nirode \\ Chemistry Department, Hofstra University, Hempstead, NY \\ *Corresponding author: Ronald.p.damelia@hofstra.edu \\ Received April 11, 2019; Revised May 14, 2019; Accepted June 01, 2019
}

\begin{abstract}
The determination of the composition of PVP-PVAc copolymers and blends is essential for knowing their polymeric properties and appropriate applications. In order to create a streamlined way to quantify the composition of PVP-PVAc copolymers and blends, the qNMR methodology alongside FTIR and elemental analysis were used to develop calibration curves for industrial use. We report on the methodologies used to determine \% PVP content in the copolymers and blends in question as well as the results obtained via NMR, FTIR, and elemental analysis. Results from the NMR analysis were corroborated with calibration curves used to determine \% PVP composition using FTIR and were further corroborated with elemental analysis results. The PVP-PVAc copolymers used ranged from 30 to $70 \mathrm{~mol} \% \mathrm{PVP}$ and the blends ranged from 20 to 80 weight $\%$ PVP.
\end{abstract}

Keywords: quantitative analysis, Nuclear Magnetic Resonance, elemental analysis, Fourier Transform Infrared Spectroscopy, n-vinyl pyrrolidone - vinyl acetate copolymer and blends

Cite This Article: Ronald P. D'Amelia, Joseph Mancuso, and William Nirode, "The Characterization of Poly n-Vinyl Pyrrolidone-Polyvinyl Acetate (PVP-PVAc) Copolymers and Blends by Nuclear Magnetic Resonance Spectroscopy, Fourier Transform Infrared Spectroscopy, and Elemental Analysis." Journal of Polymer and Biopolymer Physics Chemistry, vol. 7, no. 1 (2019): 1-9. doi: 10.12691/jpbpc-7-1-1.

\section{Introduction}

Proton Nuclear Magnetic Resonance Spectroscopy and Fourier Tranform Infrared Spectroscopy have been frequently used for qualitative and quantitative analysis of organic compounds and polymers [1-9]. However, the copolymer composition of PVP-PVAc copolymers has not been investigated thus far with proton NMR. These copolymers have a wide variety of practical applications in various industries including drug delivery, cosmetics, hair-styling, etc. [10]. PVP-PVAc copolymers have been used specifically in order to increase the dissolution of drugs that are less soluble in water than desirable such as indomethacin, tolbutamide, and nifedipine $[11,12,13,14]$. Typically these copolymers have been used to formulate the aforementioned drugs by means of hot melt extrusion as glass solutions and the results consistently show higher bioavailability [10]. PVP has been used in over 900 commercial cosmetic products [15] and PVP-PVAc copolymers are the most commonly used copolymers in the cosmetic industry, comprising over $40 \%$ of rinse-off products such as paste masks and mud packs [16]. PVP-PVAc copolymers are also frequently used in hair sprays and have been proven to be both safe and effective $[16,17,18,19]$. PVP-PVAc finds use in FDA approved food supplements as well [20]. Due to the fact that PVP-PVAc copolymer usages vary based off the copolymer composition, it is of critical importance to develop a toolset that will allow industry leaders to determine their products copolymer ratio for quality control purposes. The objective of this study was to use a variety of techniques to quantify the \% PVP in copolymers and physical blends with PVAc. In order to do this, elemental analysis, FTIR, and proton NMR have been used in order to develop calibration curves by which one can determine the copolymer composition of PVP-PVAc copolymers and physical blends. The PVP-PVAc copolymers analyzed in this study ranged from 30-70 mol\% PVP. In addition to copolymers of PVP-PVAc, blends were also analyzed via FTIR and NMR and these ranged from $20-80$ weight $\%$ PVP. 


\section{Materials and Methods}

\subsection{Materials}

For the NMR analysis of the PVP-PVAc copolymers and blends, a $400 \mathrm{MHz}$ JEOL model ECS-400 NMR spectrometer was used. The JEOL Delta NMR control and process software version 5.0.2 (Windows) were used to analyze individual spectrum. Each sample was run in deuterated chloroform without an internal reference standard as a 32 scan single pulse, 1D proton NMR with a $0.25 \mathrm{~Hz}$ resolution and a relaxation time ranging from 8 to 10 seconds. The NMR tubes used in this study were Wilmad Pyrex glass $5 \mathrm{~mm} \times$ 7" thin wall tubes. Any vendors of NMR hardware and software can be used to perform this experiment. The PVP-PVAc copolymers were obtained from Scientific Polymer Products $\left(\mathrm{SP}^{2}\right)$, Inc., Ontario, N.Y. These copolymers ranged from 30 to $70 \mathrm{~mol} \%$ PVP. The 30, 50, and 70\% copolymers were obtained as $50 \mathrm{w} / \mathrm{w} \%$ solutions in isopropanol. Each sample of PVP-PVAc copolymer was analyzed in the solid phase. Blends of PVP and PVAc were prepared by dissolving PVP homopolymers having a Mw of $40 \mathrm{~K}$ Daltons with homopolymers of PVAc having a $\mathrm{Mw}$ of $39 \mathrm{~K}$ Daltons in methanol. These solutions were evaporated in a vacuum oven at $50^{\circ} \mathrm{C}$ at $20 \mathrm{~mm}$ of $\mathrm{Hg}$ leaving solid blends of homopolymers ranging from 20 to $80 \%$ PVAc. For FTIR analysis of the PVP-PVAc copolymers and blends, a Bruker Alpha-P ${ }^{\circledR}$ FT-IR spectrometer with a Platinum ${ }^{\circledR}$ attenuated total internal reflectance (ATR) quick snap module with a diamond crystal was used. The Alpha-P unit has a Michelson Interferometer with a $\mathrm{SiC}$ globar as the IR source, the time dependence of the IR intensity is measured with a pyroelectric DTGS detector. The data analysis was done using the Bruker OPUS® software version 7.0.122. The spectra were displayed as absorbance vs frequency in wavenumbers ranging from 500 to $4000 \mathrm{~cm}^{-1}$. The spectra were done in absorbance mode since absorbance is proportional to concentration according to Beer's Law.

\subsection{Methods}

Summarized in Table 1 and Table 2 are the copolymer solutions and blend solutions prepared for NMR analysis respectively. These samples were left in $2 \mathrm{ml}$ sample vials over-night and were sealed with Teflon caps. This was done in order to maximize the dissolution of the copolymers. After dissolving over-night, the samples were then aliquotted into NMR tubes in $400 \mathrm{ul}$ increments using a p1000 Gilson Classic pipette. Approximately 1.000 grams of each blend used in this study were prepared by weighing PVP and PVAc homopolymers in the amounts shown in Table 3. For FTIR analysis, the PVP-PVAc samples were analyzed with no sample preparation other than being pressed between the anvil and the diamond crystal of the ATR unit in the solid phase. The samples were measured using 16 scans at a resolution of $4 \mathrm{~cm}^{-1}$. The elemental analysis of the PVP-PVAc copolymers was done via Galbraith Laboratories in Knoxville Tennessee and the $\% \mathrm{C}, \% \mathrm{H}$, and $\% \mathrm{~N}$ were obtained to determine $\%$ PVP.

Table 1. Summary of NMR solutions of PVP-PVAc Copolymers

\begin{tabular}{|c|c|c|}
\hline Nominal mole\% PVP & Weight $(\mathrm{mg})$ & Volume of $\mathrm{CDCl}_{3}(\mathrm{ml})$ \\
\hline 30 & 22.2 & 1.00 \\
\hline 50 & 20.1 & 1.00 \\
\hline 60 & 21.3 & 1.00 \\
\hline 70 & 21.6 & 1.00 \\
\hline
\end{tabular}

Table 2. Summary of NMR solutions of PVP-PVAc Blends

\begin{tabular}{|c|c|c|}
\hline Gravimetric weight \% PVP & Weight $(\mathrm{mg})$ & Volume of $\mathrm{CDCl}_{3}(\mathrm{ml})$ \\
\hline 20 & 18.9 & 1.00 \\
\hline 40 & 21.3 & 1.00 \\
\hline 50 & 20.6 & 1.00 \\
\hline 60 & 18.8 & 1.00 \\
\hline 80 & 19.2 & 1.00 \\
\hline
\end{tabular}

Table 3. Summary of PVP-PVAc blend composition

\begin{tabular}{|c|c|c|}
\hline Weight of PVP $(\mathrm{g})$ & Weight of PVAc $(\mathrm{g})$ & Weight \% PVP \\
\hline .8046 & .1867 & 81.17 \\
\hline .6005 & .4005 & 59.99 \\
\hline .5140 & .4862 & 50.39 \\
\hline .4025 & .5961 & 40.31 \\
\hline .2013 & .8005 & 20.09 \\
\hline
\end{tabular}

\subsection{Hazards}

Persons with metallic implants should not be near the NMR as the strong magnetic field poses a danger to people with metallic implants and pacemakers. Deuterated chloroform is acutely toxic orally, dermally, and via inhalation, it is also a suspected carcinogen as such the use of a safety hood, goggles, and gloves are required. Methanol is highly flammable and toxic if inhaled, thus use of a fume hood is required.

\subsection{Statistical Analysis}

NMR samples were run five times and the data reported is the average \% PVP determined via the calibration curve. FTIR analysis was run eight times and the data reported is the average. Elemental analysis data was reported as the average $\% \mathrm{C}, \% \mathrm{H}$, and $\% \mathrm{~N}$ determined via duplicate analyses. The standard deviations ranged from 0.259 to 0.0068 .

\section{Results and Discussion}

\subsection{NMR Analysis}

Figure 1 through Figure 4 are the NMR spectra of PVP homopolymer, PVAc homopolymer, 30\% PVP-PVAc Copolymer, and 50\% PVP-PVAc Blend respectively. 


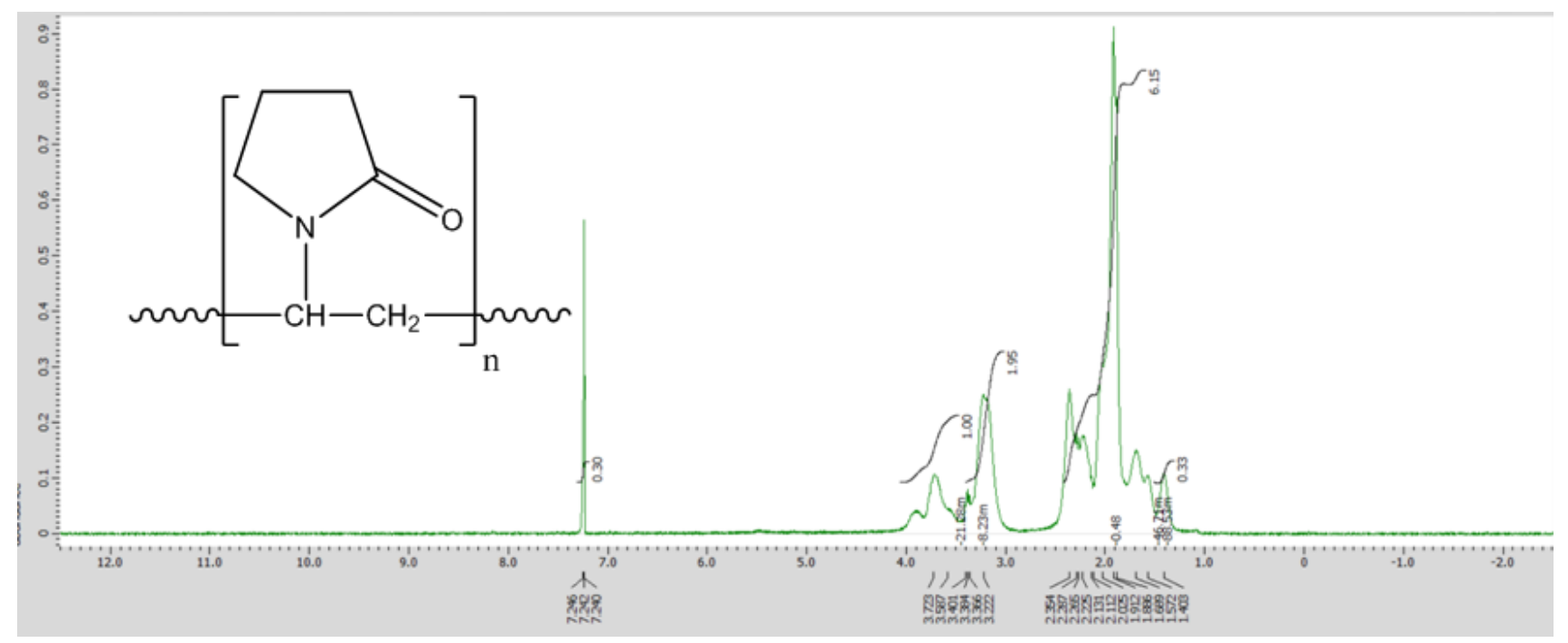

Figure 1. Proton NMR Spectrum of the PVP homopolymer in $\mathrm{CDCl}_{3}$

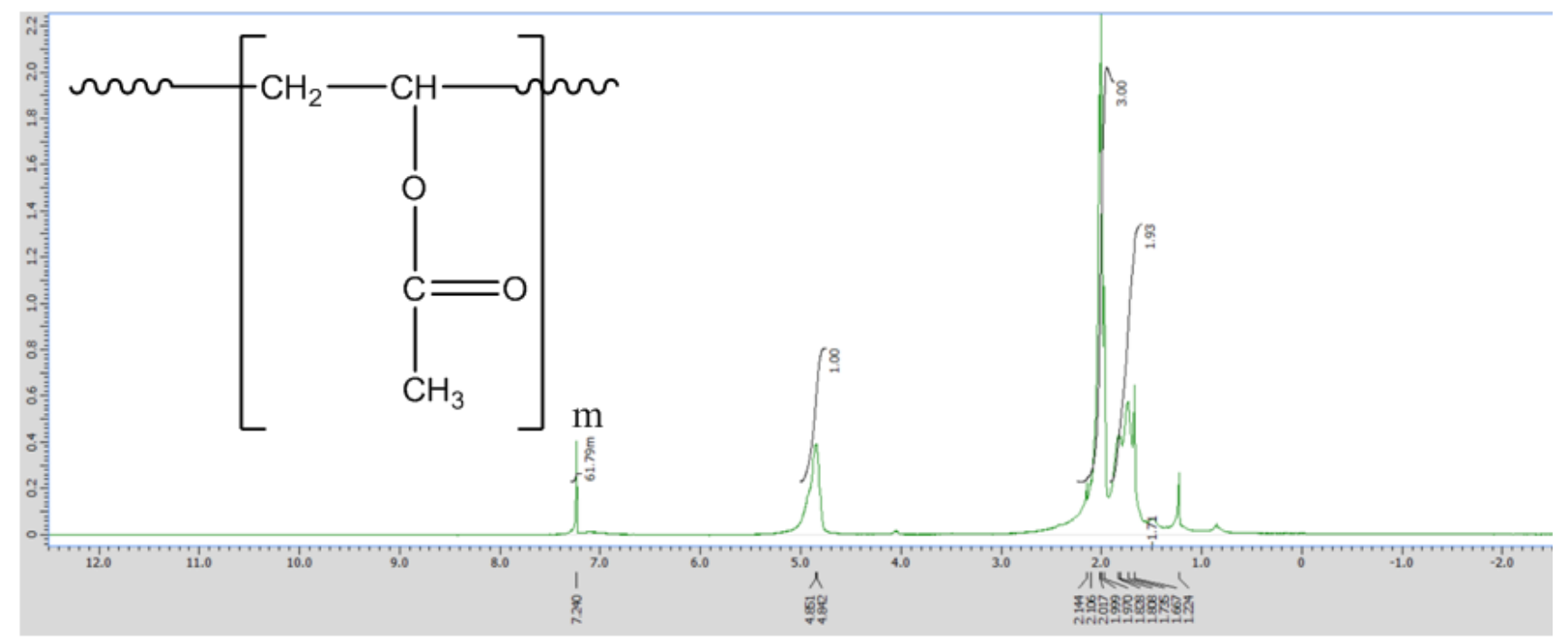

Figure 2. Proton NMR Spectrum of the PVAc homopolymer in $\mathrm{CDCl}_{3}$

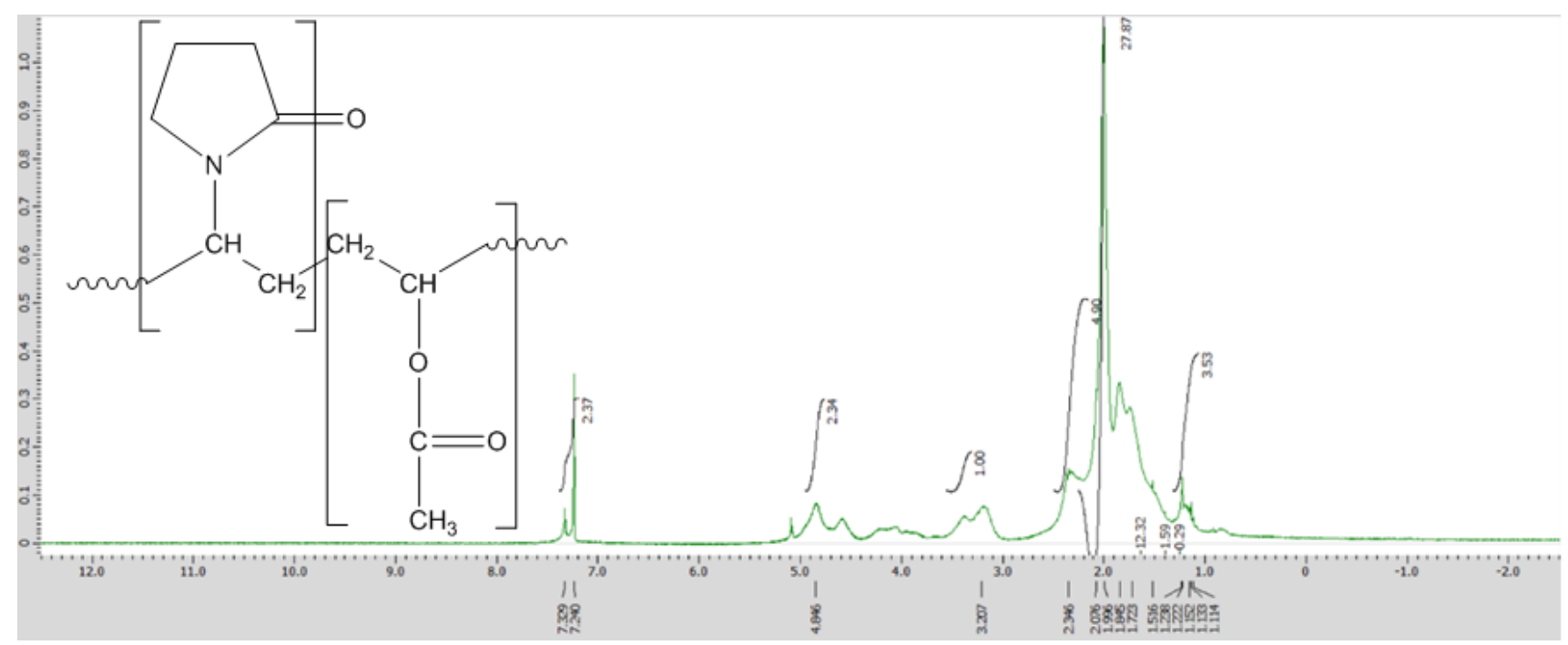

Figure 3. Proton NMR spectrum of the $30 \mathrm{~mol} \% \mathrm{PVP}$, PVP-PVAc Copolymer in $\mathrm{CDCl}_{3}$ 


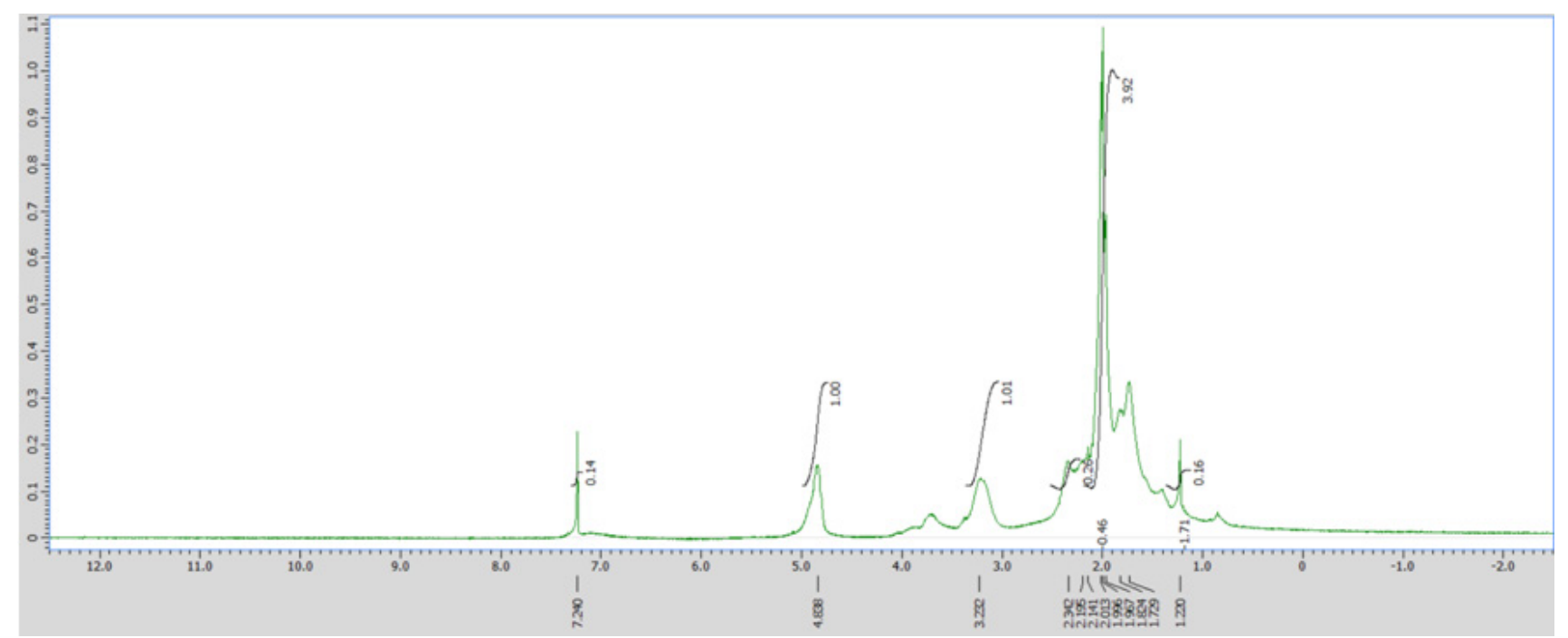

Figure 4. Proton NMR spectrum of the 50 weight $\%$ PVP, PVP-PVAc Blend in $\mathrm{CDCl}_{3}$

All peaks at $7.2 \mathrm{ppm}$ are due to the chloroform solvent, this peak is far downstream due to the highly de-shielding chemical environment the proton of the chloroform experiences because of the extremely electronegative chlorine atoms attached to the carbon. The NMR spectrum of PVP shows that there are peaks at $3.7 \mathrm{ppm}, 3.2 \mathrm{ppm}$, and from 2.5 to $1.4 \mathrm{ppm}$. The $3.7 \mathrm{ppm}$ peak is associated with the methyne proton underneath the pyrrolidone ring. This is due to the highly polar nature of the carbon-nitrogen bond as well as the electron withdrawing effects of the carbonyl carbon in the pyrrolidone ring. These two factors create the most de-shielded environment of all the protons involved. The $3.2 \mathrm{ppm}$ peak is associated with the methylene proton of the carbon adjacent to the methyne carbon previously mentioned. These protons are located upstream of the methyne protons due to them being located farther away from the carbonyl group in the pyrrolidone ring as well as farther away from the carbonnitrogen bond. The series of peaks from 2.5 to $1.4 \mathrm{ppm}$ are all associated with the six protons in the pyrrolidone ring as they are the least de-shielded of all the protons involved in the structure since the electron withdrawing effects of the carbonyl carbon and carbon-nitrogen bond, in essence, cancel each other out to a more appreciable extent for the protons in the ring. The NMR spectrum of PVAc show peaks at $4.8 \mathrm{ppm}, 2.1 \mathrm{ppm}$, and $1.8 \mathrm{ppm}$. The $4.8 \mathrm{ppm}$ peak corresponds to the methyne proton above the oxygen of the acetate moiety. This is due to the polar nature of the carbon-oxygen bond as well as the electron withdrawing effects of the carbonyl group. This chemical environment is the most de-shielding and therefore the proton would resonate downstream of the others. The peak at $2.1 \mathrm{ppm}$ corresponds to the terminal methyl group adjacent to the carbonyl carbon. The carbonyl carbon creates a moderately de-shielding environment for the methyl protons and therefore they resonate downstream of the protons in the backbone. The polar bond between the carbon and oxygen is not present in the terminal methyl group and therefore it resonates upstream of the methyne proton. The peak at 1.8 ppm corresponds to the methylene protons present in the backbone of the PVAc homopolymer, these protons are not subject to polar bonds nor are they close enough to the carbonyl carbon to resonate further downstream, though the acetate moiety does cause some de-shielding leading them to resonate closer to 2 rather than 1 ppm. Table 4 summarizes the critical peaks associated with PVP and PVAc including their normalized integration value as well as their chemical shift value.

Table 4. Summary of key 1 H NMR peaks for PVP and PVAc homopolymer

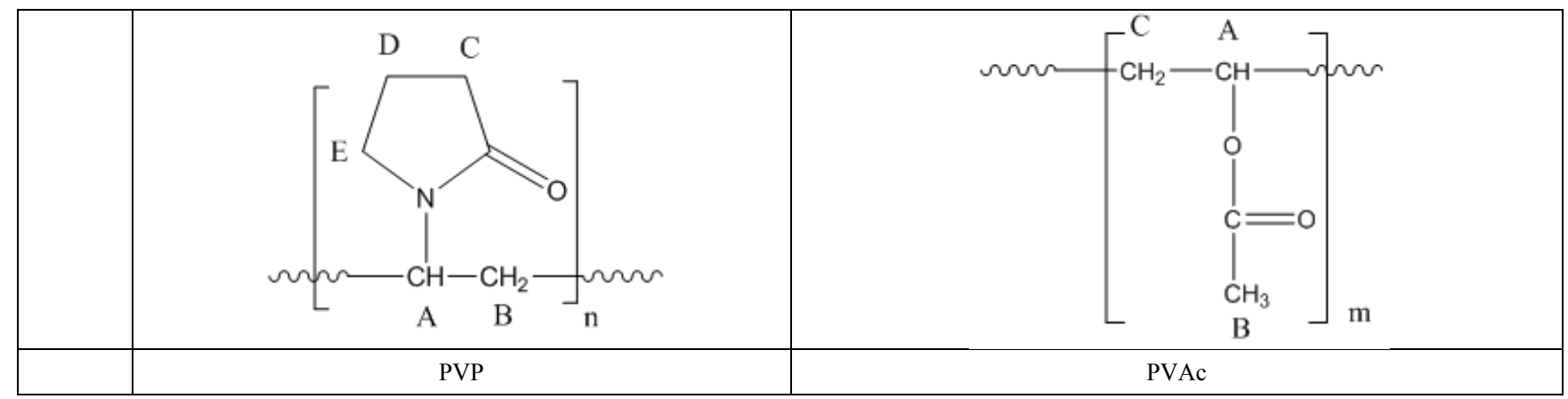

homopolymer

\begin{tabular}{|c|c|c|c|c|c|}
\hline Protons & Chemical shift value (ppm) & Normalized Integration value & Protons & Chemical shift value (ppm) & Normalized Integration value \\
\hline A & 3.7 & 1.00 & A & 4.8 & 1.00 \\
\hline B & 3.2 & 1.95 & B & 2.1 & 3.00 \\
\hline C, D, E & $2.5-1.4$ & 6.15 & C & 1.8 & 1.93 \\
\hline
\end{tabular}


In order to determine \% PVP in the PVP-PVAc copolymers and blends, the following equation was used:

$$
\% P V P=100 * \frac{\# \text { of methyne PVP protons }}{\# \text { of methyne PVP and PVAc protons }} .
$$

Table 5 summarizes the weight \% PVP determined by NMR and the actual weight \% PVP for the copolymers. Table 6 summarizes the weight \% PVP determined via the calibration curve versus the actual weight \% PVP for copolymers. Figure 5 shows the calibration curve relating qNMR determined weight \% PVP versus actual weight \% PVP with error bars representing a 95\% confidence interval using a student's $t$ distribution with $n$ $=5$ and 4 degrees of freedom. The relationship is decidedly linear, with a coefficient of determination greater than 0.999 .

Table 5. Summary of qNMR Determined Weight \% PVP vs Actual Weight \% PVP Derived From Nominal Mole\%

\begin{tabular}{|l|l|}
\hline qNMR weight \% PVP & Actual weight \% PVP \\
\hline 34.55 & 33.65 \\
\hline 47.13 & 47.30 \\
\hline 49.05 & 49.85 \\
\hline 58.15 & 60.09 \\
\hline
\end{tabular}

Table 6. Summary of Calibration Curve Weight \% PVP vs Actual Weight \% PVP Derived From Nominal Mole\%

\begin{tabular}{|l|l|}
\hline Calibration curve weight \% PVP & Actual weight \% PVP \\
\hline 34.65 & 33.65 \\
\hline 46.84 & 47.30 \\
\hline 49.11 & 49.85 \\
\hline 58.25 & 60.09 \\
\hline
\end{tabular}

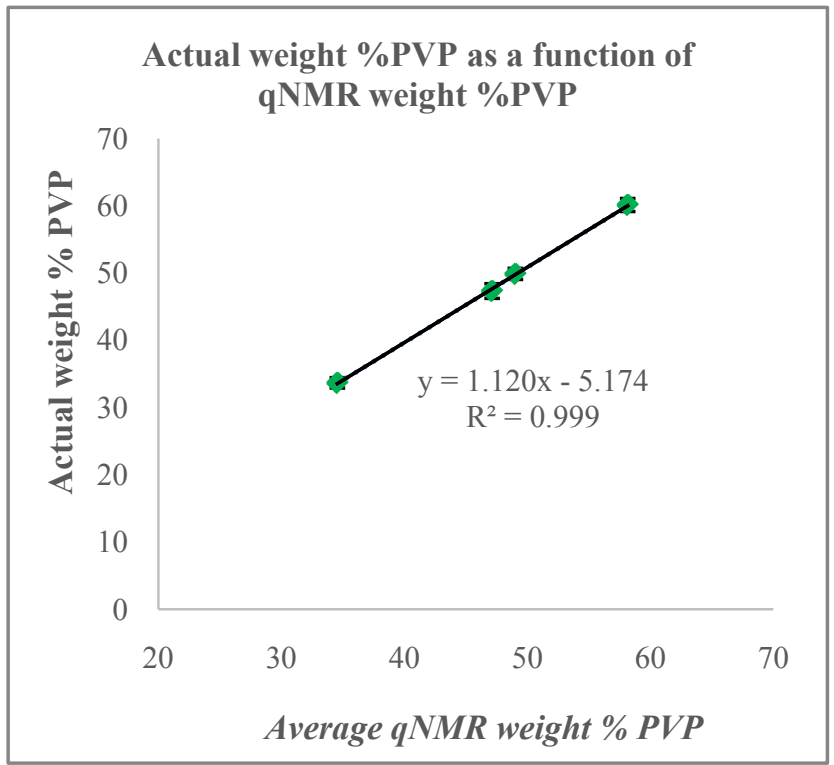

Figure 5. actual weight \% PVP plotted against average qNMR weight $\%$ PVP in copolymers with PVAc. Error bars represent 95\% CI with $n=5$

Table 7 showcases the qNMR weight \% PVP in blends with PVAc versus actual weight \% PVP. Table 8 indicates the calibration curve determined weight \% PVP in blends with PVAc versus actual weight \% PVP. Figure 6 shows the calibration curve for the PVP-PVAc blends and plots the actual weight $\%$ PVP versus the average qNMR determined weight $\%$ PVP. The curve displays an extremely linear relationship, having an $\mathrm{R}^{2}$ value greater than 0.999 .

Table 7. Summary of qNMR Weight \% PVP and Actual Weight \% PVP in Blends with PVAc

\begin{tabular}{|l|l|}
\hline qNMR weight \% PVP & Actual weight \% PVP \\
\hline 79.66 & 81.17 \\
\hline 59.86 & 59.99 \\
\hline 50.18 & 51.39 \\
\hline 39.10 & 40.31 \\
\hline 20.00 & 20.09 \\
\hline
\end{tabular}

Table 8. Summary of Calibration Curve Determined Weight \% PVP and Actual Weight \% PVP in Blends with PVAc

\begin{tabular}{|l|l|}
\hline Calibration curve weight \% PVP & Actual weight \% PVP \\
\hline 79.87 & 81.17 \\
\hline 59.02 & 59.99 \\
\hline 50.55 & 51.39 \\
\hline 39.64 & 40.31 \\
\hline 19.73 & 20.09 \\
\hline
\end{tabular}

Gravimetric weight \% PVP as a function of qNMR weight $\%$ PVP

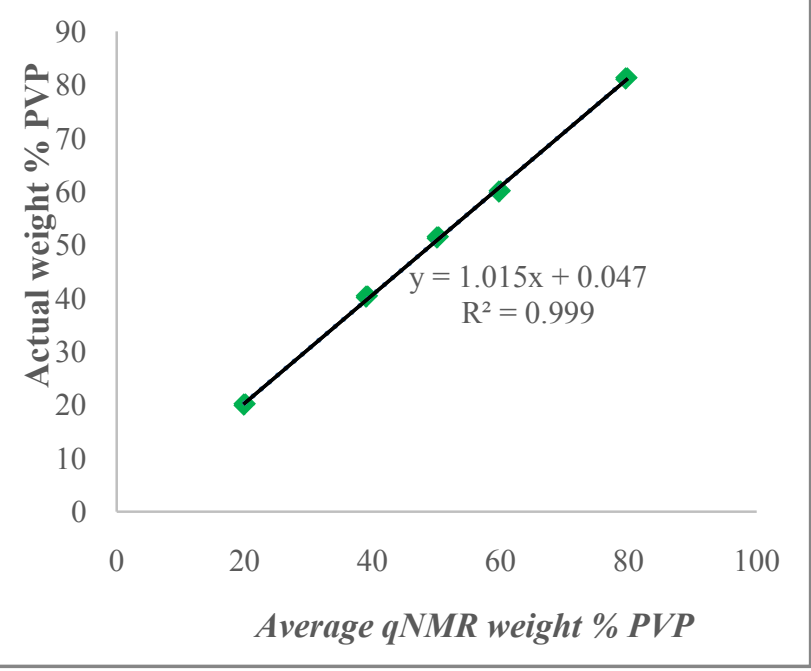

Figure 6. Actual weight \% PVP plotted against average qNMR weight $\%$ PVP in blends with PVAc

\subsection{FTIR Analysis}

Figure 7 through Figure 9 showcase the FTIR spectra of the PVP homopolymer, the PVAc homopolymer, and a 50\% PVP, PVP-PVAc copolymer respectively.

The PVP homopolymer displays an absorbance peak at $1670 \mathrm{~cm}^{-1}$ due to it containing a carbonyl carbon and this is the $\mathrm{C}=\mathrm{O}$ stretch vibrational mode. The PVAc homopolymer displays an absorbance peak at $1230 \mathrm{~cm}^{-1}$ 
due to its ester functionality, this peak corresponds to a C$\mathrm{O}$ stretching between the methyne carbon and the oxygen adjacent to the carbonyl carbon. These are the two peaks that are used to determine \% PVP in the copolymers and blends since they are unique identifiers of the level of PVP and PVAc. The PVP carbonyl peak absorbs infrared radiation at lower wavenumbers than the PVAc carbonyl peak and therefore by taking the area of the strong peak at $1670 \mathrm{~cm}^{-1}$ and dividing it by the area of the strong peak at $1230 \mathrm{~cm}^{-1}$ it is possible to gauge \% PVP levels in the copolymer by relating the ratio and the known \% PVP present. Table 9 shows the FTIR integration ratio between the $1670 \mathrm{~cm}^{-1}$ tagged for PVP and the $1230 \mathrm{~cm}^{-1}$ peak tagged for PVAc. In all cases, as the \% PVP increased in the sample, so too did the observed ratio. Figures 10 and 11 showcase the calibration curves developed by relation nominal mole \% PVP to the absorbance ratio and by relating the experimentally determined weight \% PVP to the absorbance ratio. Both relations show error bars representing one standard deviation from the mean with $\mathrm{n}=8$. Both graphs show a strong linear correlation, having $\mathrm{R}^{2}$ values greater than 0.99 .

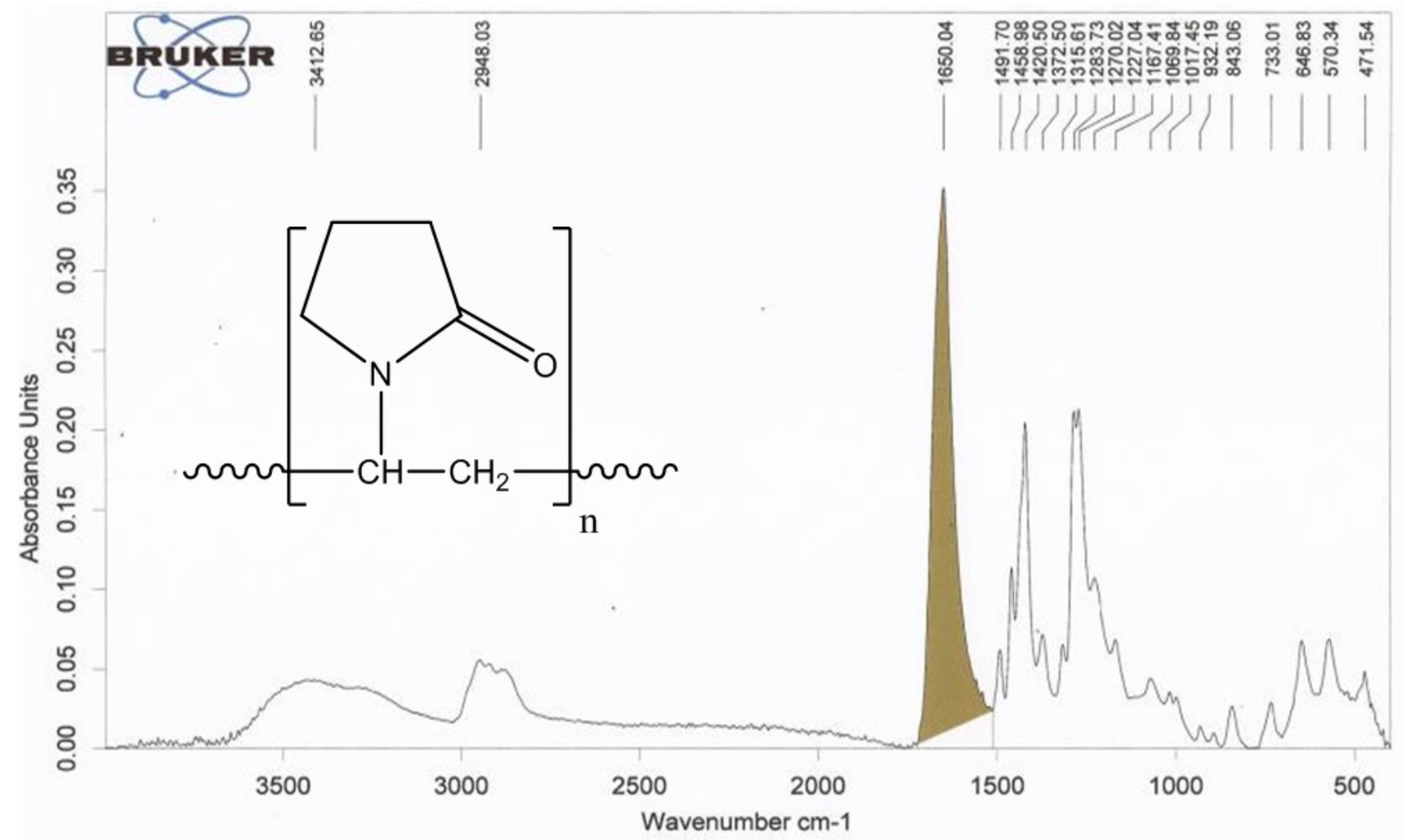

Figure 7. FTIR spectrum of PVP homopolymer. The major characteristic PVP Absorbance bandwas located at $\underline{1650} \mathrm{~cm}^{-1}$

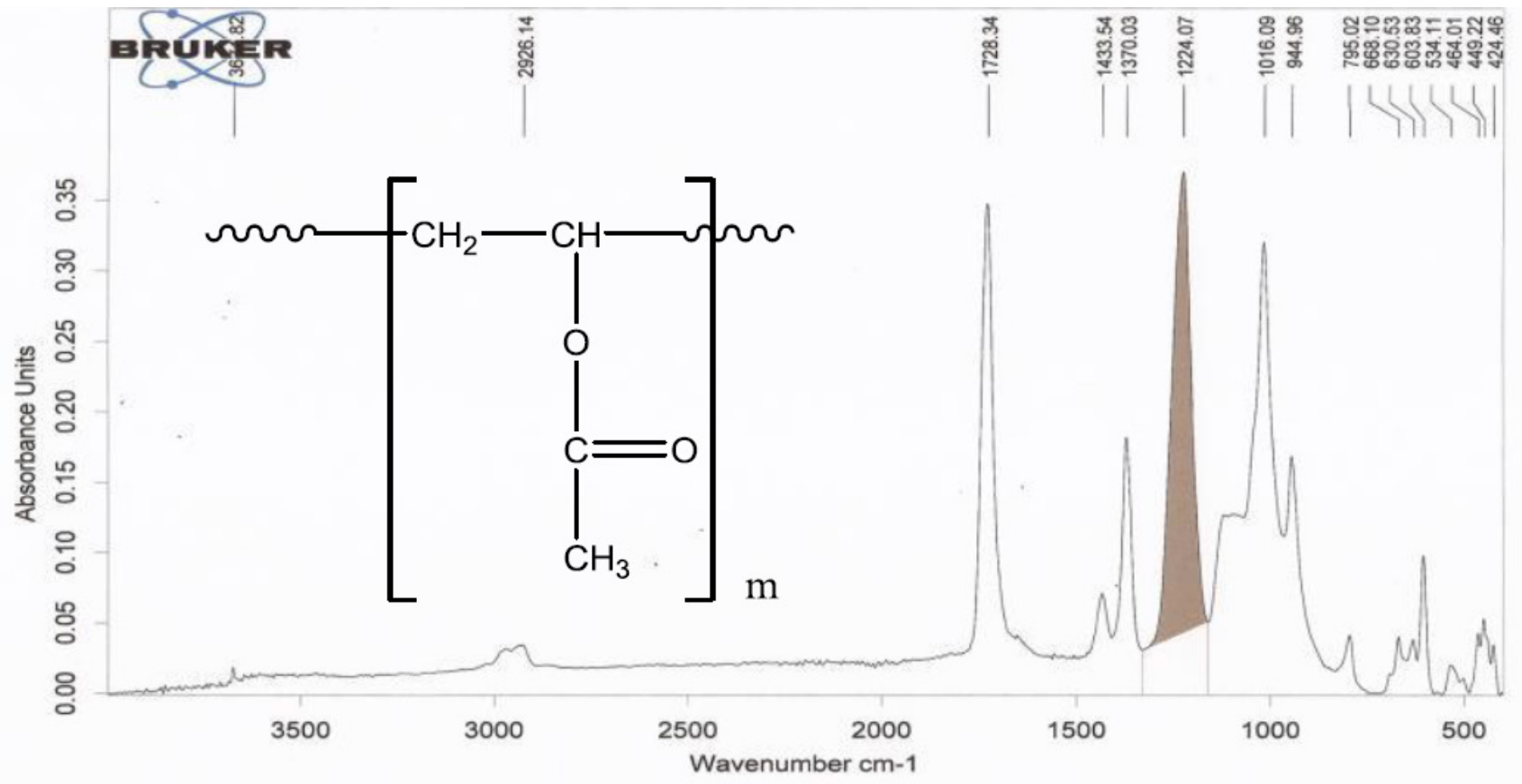

Figure 8. The FTIR spectrum of PVAc key bands are at $1728 \mathrm{~cm}^{-1}, 1433 \mathrm{~cm}^{-1}, 1370 \mathrm{~cm}^{-1}, \underline{1224} \mathrm{~cm}^{-1}$, and $1016 \mathrm{~cm}^{-1}$ 


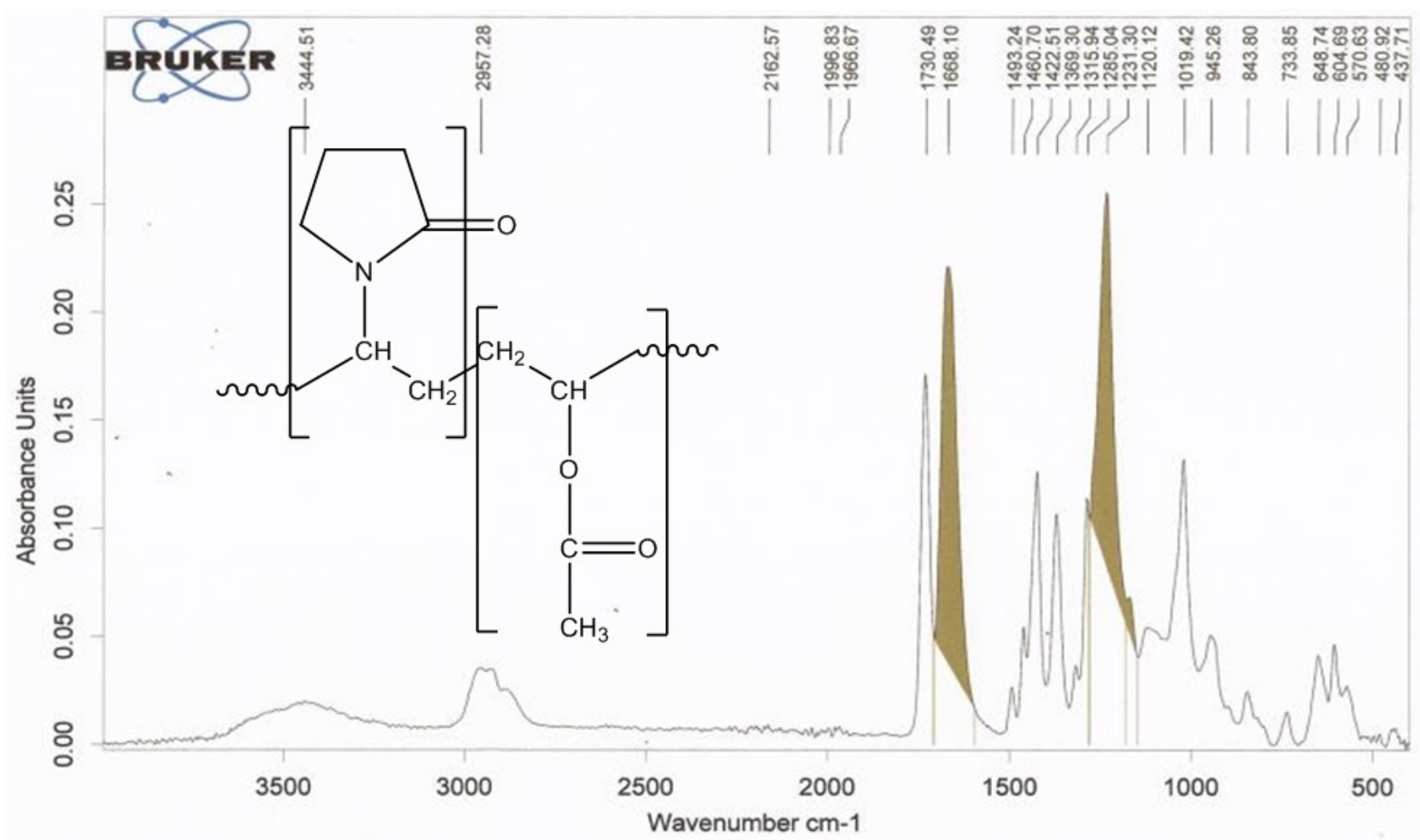

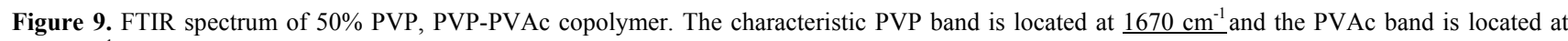
$\underline{1230 \mathrm{~cm}^{-1}}$

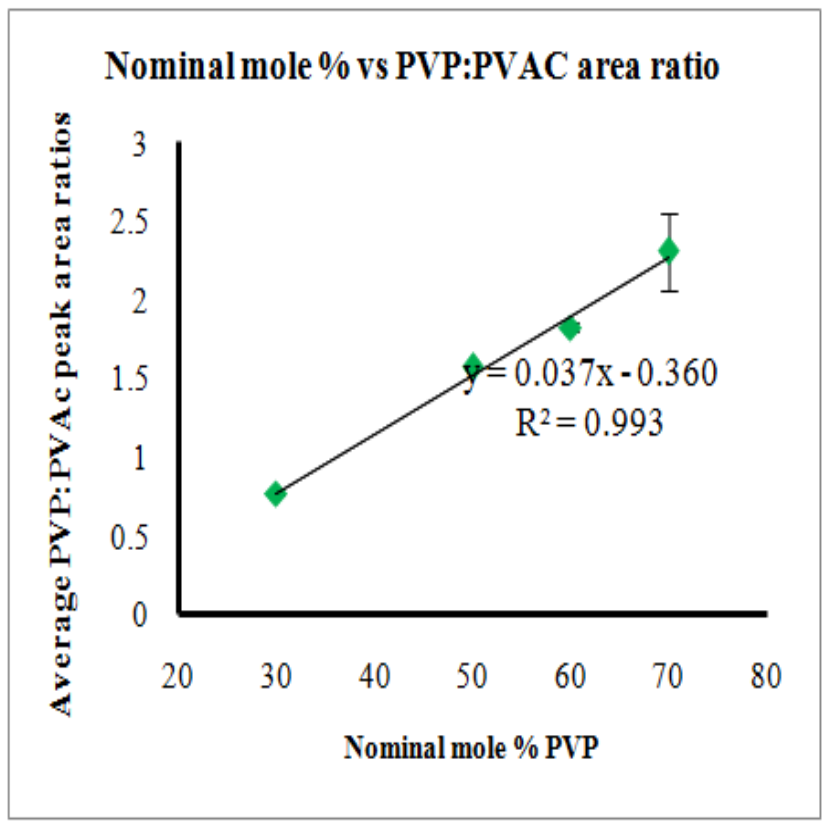

Figure 10. Nominal mole \% PVP plotted versus average PVP:PVAc peak area ratios

Table 9. Summary of FTIR Integration Ratios for PVP-PVAc Copolymers

\begin{tabular}{|c|c|c|}
\hline $\begin{array}{c}\text { Weight \% PVP } \\
\text { (Experimental) }\end{array}$ & $\begin{array}{c}\text { Mole \% PVP } \\
\text { (Nominal) }\end{array}$ & $\begin{array}{c}\left.\text { PVP(1670 } \mathrm{cm}^{-1}\right): \text { PVAc(1230cm } \\
\text { peak area ratio }\end{array}$ \\
\hline 60.09 & 70 & 2.30 \\
\hline 49.85 & 60 & 1.82 \\
\hline 47.30 & 50 & 1.57 \\
\hline 33.65 & 30 & 0.76 \\
\hline
\end{tabular}

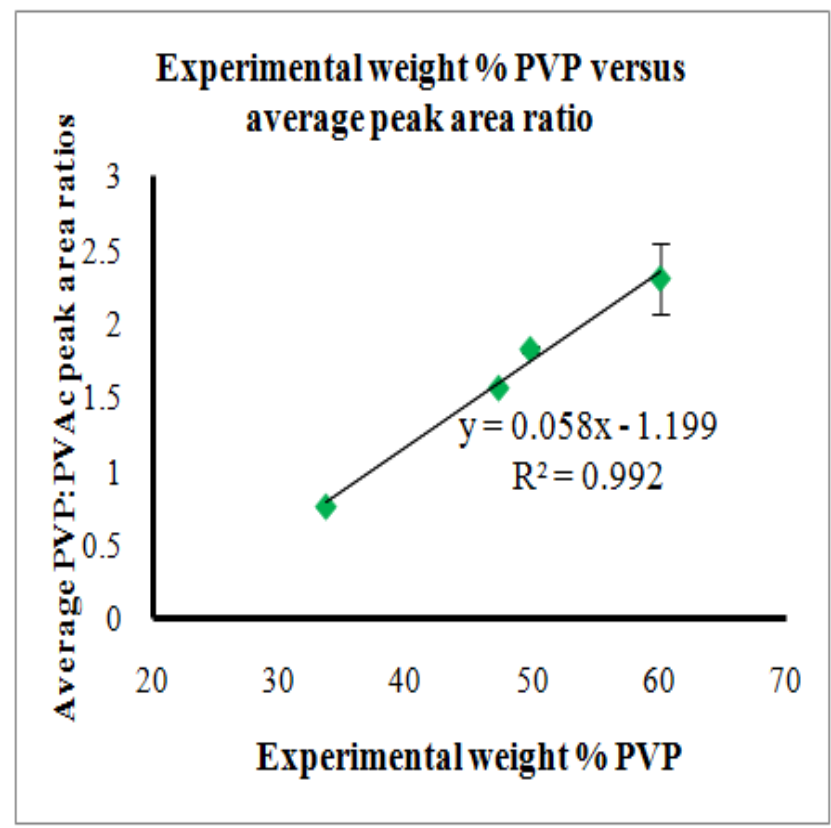

Figure 11. Experimental weight \% PVP plotted versus average PVP:PVAc peak area ratios

Table 10. Summary of Average FTIR Ratios for Blends of PVPPVAc

\begin{tabular}{|l|l|}
\hline Weight \% PVP & PVP:PVAc peak area ratio \\
\hline 81.17 & 2.71 \\
\hline 59.99 & 2.01 \\
\hline 50.39 & 1.59 \\
\hline 40.31 & 1.17 \\
\hline 20.09 & 0.45 \\
\hline
\end{tabular}


Table 10 shows the FTIR ratios between the PVP peak and the PVAc peak with relation to the weight \% PVP in PVP-PVAc blends. Figure 12 shows the calibration curve developed by running FTIR analyses of each sample eight times. Error bars represent one standard deviation from the mean. In all cases the ratio increased as the weight \% PVP increase and is shown to be a very strong linear correlation, having a coefficient of determination greater than 0.99 .

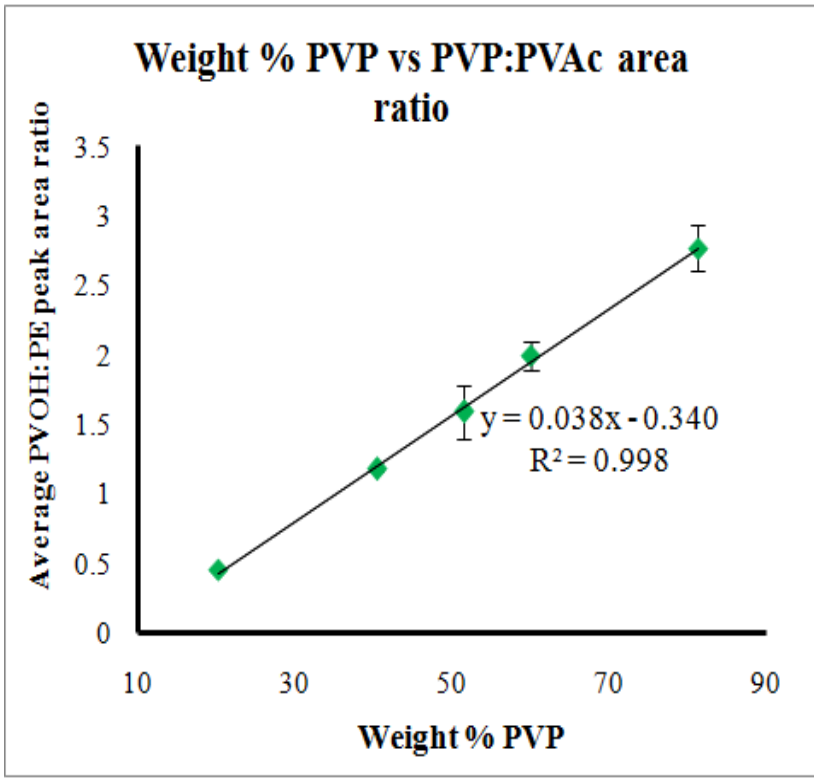

Figure 12. Experimental weight $\%$ PVP plotted versus average PVP:PVAc peak area ratios

\subsection{Elemental Analysis}

The experimental weight \% PVP was determined via elemental analysis, the results are described in Table 11 which includes \% Carbon, \% Hydrogen, \%Nitrogen, and $\%$ Oxygen which was obtained by difference. In order to determine the exact \% PVP, the following equation was used:

$$
\% P V P=(\% N) / 12.61 * 100 \%
$$

Table 11. Summary of Elemental Analysis Results for PVP-PVAc Copolymers.

\begin{tabular}{|c|c|c|c|c|c|c|}
\hline 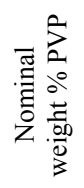 & 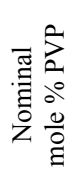 & 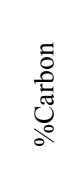 & 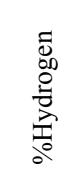 & 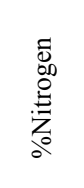 & 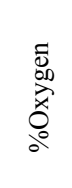 & 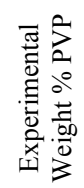 \\
\hline 60.74 & 70 & 60.37 & 8.083 & 7.659 & 23.89 & 60.09 \\
\hline 55.19 & 60 & 58.5 & 7.67 & 6.959 & 26.87 & 49.85 \\
\hline 49.85 & 50 & 59.05 & 7.743 & 6.239 & 26.97 & 47.30 \\
\hline 34.73 & 30 & 58.31 & 7.45 & 4.379 & 29.86 & 33.65 \\
\hline
\end{tabular}

Figure 13 shows the relationship between the experimentally determined weight \% PVP versus the nominal weight \% PVP which was obtained via converting the nominal mole \% PVP to weight \%. In all cases, the experimental weight \% PVP increased as the nominal weight \% PVP increased. The relationship is linear with an $\mathrm{R}^{2}$ value greater than 0.96 .

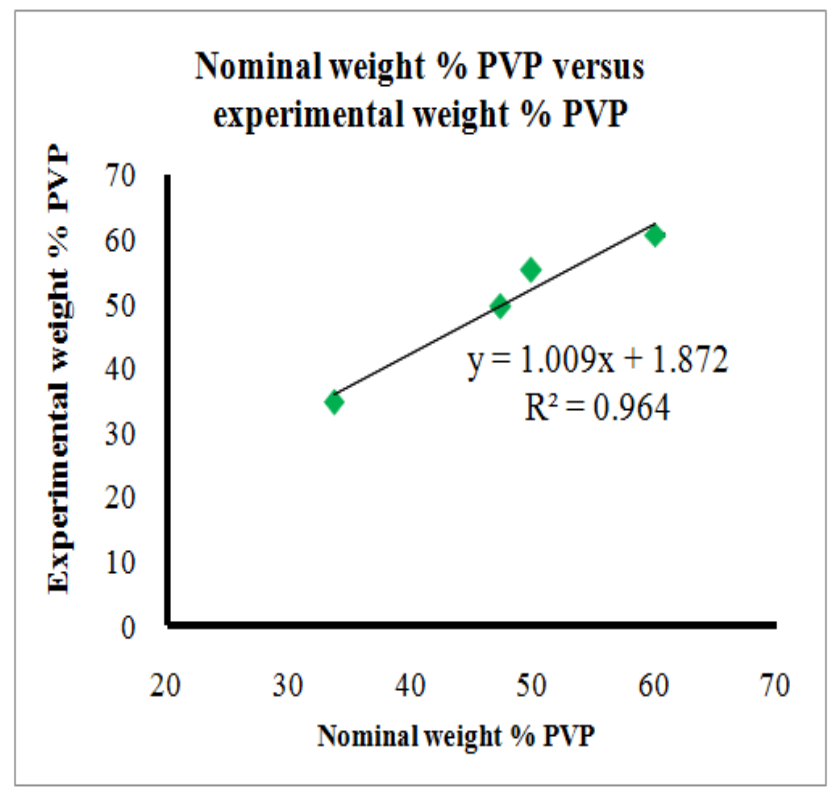

Figure 13. Nominal weight \% PVP plotted versus experimental weight $\%$ PVP

\section{Conclusions}

1H NMR, FTIR, and Elemental Analysis are indispensable tools for determining copolymer composition of PVPPVAc copolymers, they can be used in isolation or in tandem in order to get an accurate depiction of the \% PVP contained within the copolymer. The most accurate method is $1 \mathrm{H}$ NMR which showed the highest degree of linearity as well as the highest specificity compared to the other methods shown. The methodology used in this study can be readily adapted for industrial use in all applicable areas, as all the methods utilized are high throughput quality control mechanisms. NMR, FTIR, and Elemental Analysis all align very closely. The above analysis serves as evidence of the quantitative utility of these tools in an industrial setting.

\section{Acknowledgments}

We acknowledge the support from Hofstra University HCLAS Faculty Research and Development Grant.

\section{Statement of Competing Interests}

The Authors have no competing interests.

\section{List of Abbreviations}

PVP - Polyvinyl pyrrolidone

PVAc - Polyvinyl acetate

FTIR- Fourier transform infrared spectroscopy

NMR- Nuclear Magnetic Resonance Spectroscopy

$1 \mathrm{H}$ NMR- The same as proton NMR 


\section{References}

[1] Gebel, M.E., Kaleuati, M. A., Finlayson-Pitts, B. J. "Measurement of the Organics using Three FTIR Techniques: Absorption, Attenuated Total Reflectance, and Diffuse Reflectance", J. Chem. Ed., 80 (6), 672-675, June 2003.

[2] Lutz, J, Matyjaszewski, K "Nuclear Magnetic Resonance Monitoring of Chain-End Functionality in the Atom Transfer Radical Polymerization of Styrene", J. Polymer Sci. January 2005.

[3] Veening, H., "Quantitative Infrared Analysis of Xylene Mixtures: Internal Standard Method", J. Chem. Ed., 46 (6), 319-320, June 1966.

[4] Hu, W, Mao, J, Schmidt-Rohr, K, Xing, B "Poly(methylene) Crystallites in Humic Substances Detected by Nuclear Magnetic Resonance" Environ. Sci. Technol. December 1999.

[5] Silverstein, R.M., Webster F.X., Spectrometer Identification of Organic Compounds, 7th edition, Wiley \& Sons, New Jersey, December 2011.

[6] Robinson J.W., Frame E.M.S., Frame 11 G.M., Undergraduate Instrumental Analysis, 7th edition, CRC Press -Taylor Francis, Boca Raton, Florida, chapter 4, July 2014

[7] Schuttlefield, J.D.; Grassian, V.H., "ATR-FTIR Spectroscopy in the Undergraduate Chemistry Laboratory", J. Chem. Educ., 85 (2), 279-281, February 2008.

[8] Lambert J.B., Gronert S., Shurvell H.F., Lightner D., Organic Structural Spectroscopy, 2nd, edition, Prentice Hall, New Jersey, October 2010.

[9] Smith iv, R.E., McKee, J.R., Zanger, M. "The Electrophilic Bromination of Toluene: Determination of the Ortho, Meta, Para, ratios by Quantitative FTIR Spectroscopy", J. Chem. Ed., 79 (2), 227-229, February 2002.

[10] Betageri, G, Kadajji, V, "Water Soluble Polymers for Pharmaceutical Applications" Polymers, 3 November 2011

[11] Forster, A, Hempenstall, J, Rades, T "Characterization of Glass Solutions of Poorly Water-Soluble Drugs Produced by Melt
Extrusion with Hydrophilic Amorphous Polymers." J. Pharm. Pharmcol. January 2001

[12] Jijun, F, Lishuang, X, Xioli, W, Shu, Z, Xiaoguang, T, Xingna, Z, Haibing, H, Xing, T "Nimodipine (NM) Tablets with High Dissolution Containing NM Solid Dispersions Prepared by HotMelt Extrusion.” Drug Dev. Ind. Pharm. March 2011

[13] He, H, Yang, R, Tang, X "In Vitro and in Vivo Evaluation of Fenofibrate Solid Dispersion Prepared by Hot-Melt Extrusion." Drug Dev. Ind. Pharm May 2010

[14] Chokshi, R.J., Sandhu, H.K., Iyer, R.M., Shah, N.H, Malick, W.A. $\mathrm{Zia}, \mathrm{H}$ "Characterization of Physico-Echanical Properties of Indomethacin and Polymers to Assess their Suitability for HotMelt Extrusion Process as a Means to Manufacture Solid Dispersion/Solution" J. Pharm. Sci. November 2005

[15] U.S. Food and Drug Administration Center for Food Safety \& Applied Nutrition (CFSAN). Voluntary Cosmetic Registration Program - Frequency of Use of Cosmetic Ingredients. College Park, MD, 2018. Obtained under the Freedom of Information Act from CFSAN; requested as "Frequency of Use Data" January 3 2018; received February 5 2018).

[16] Personal Care Products Council. 2017. Concentration of use by FDA product category - Vinylpyrrolidone Polymers. Unpublished data submitted by the Personal Care Products Council on October $2,2017$.

[17] Rothe H, Fautz R, Gerber E, Neumann L, Rettinger K, Schuh W, and Gronewold C. "Special aspects of cosmetic spray safety evaluations: Principles on inhalation risk assessment." Toxicol Lett. 2011;205(2):97-104

[18] Bremmer HJ, Prud'homme de Lodder LCH, and van Engelen JGM "Cosmetics Fact Sheet: To assess the risks for the consumer"; Updated version for ConsExpo 4. 20200

[19] Johnsen MA. "The Influence of Particle Size. Spray Technology and Marketing." 2004;14(11):24-27.

[20] EFSA Panel on Food Additives and Nutrient Sources Added to Food (ANS). "Scientific opinion on the safety of polyvinylpyrrolidone-vinyl acetate copolymer for the proposed uses as a food additive.” January 2010, EFSA Journal. 